\title{
24-h movement behaviors from infancy to preschool: cross-sectional and longitudinal relationships with body composition and bone health
}

Rachael W. Taylor ${ }^{1 *}$, Jillian J. Haszard ${ }^{1}$, Kim A. Meredith-Jones', Barbara C. Galland², Anne-Louise M. Heath³, Julie Lawrence ${ }^{2}$, Andrew R. Gray ${ }^{4}$, Rachel Sayers², Maha Hanna² and Barry J. Taylor ${ }^{5}$

\begin{abstract}
Background: New physical activity guidelines for children address all movement behaviors across the 24-h day (physical activity, sedentary behavior, sleep), but how each component relates to body composition when adjusted for the compositional nature of 24-h data is uncertain.

Aims: To i) describe 24-h movement behaviors from 1 to 5 years of age, ii) determine cross-sectional relationships with body mass index (BMI) z-score, iii) determine whether movement behaviors from 1 to 5 years of age predict body composition and bone health at 5 years.

Methods: 24-h accelerometry data were collected in 380 children over 5-7 days at 1, 2, 3.5 and 5 years of age to determine the proportion of the day spent: sedentary (including wake after sleep onset), in light (LPA) and moderate-to-vigorous physical activity (MVPA), and asleep (including naps). BMI was determined at each age and a dual-energy $x$-ray absorptiometry (DXA) scan measured fat mass, bone mineral content (BMC) and bone mineral density (BMD) at 5 years of age. 24-h movement data were transformed into isometric log-ratio co-ordinates for multivariable regression analysis and effect sizes back-transformed.

Results: At age 1, children spent $49.6 \%$ of the 24 -h day asleep, $38.2 \%$ sedentary, $12.1 \%$ in LPA, and $0.1 \%$ in MVPA, with corresponding figures of 44.4, 33.8, 19.8 and $1.9 \%$ at 5 years of age. Compositional time use was only related significantly to BMl z-score at 3.5 years in cross-sectional analyses. A 10\% increase in mean sleep time (65 min) was associated with a lower $\mathrm{BMI} z$-score (estimated difference, $-0.25 ; 95 \% \mathrm{Cl},-0.42$ to -0.08 ), whereas greater time spent sedentary $(10 \%, 47 \mathrm{~min})$ or in LPA (10\%, $29 \mathrm{~min})$ were associated with higher BMI z-scores $(0.12$ and 0.08 respectively, both $p<0.05)$. Compositional time use from 1 to 3.5 years was not related to future BMI $z$-score or percent fat. Although MVPA at 2 and 3.5 years was consistently associated with higher BMD and BMC at 5 years, actual differences were small.
\end{abstract}

Conclusions: Considerable changes in compositional time use occur from 1 to 5 years of age, but there is little association with adiposity. Although early MVPA predicted better bone health, the differences observed had little clinical relevance.

Trial registration: ClinicalTrials.gov number NCT00892983.

Keywords: Physical activity, Sedentary behavior, Sleep, Children, Compositional time use

\footnotetext{
*Correspondence: rachael.taylor@otago.ac.nz

'Departments of Medicine, Dunedin School of Medicine, University of Otago,

PO Box 56, Dunedin, New Zealand

Full list of author information is available at the end of the article
}

(c) The Author(s). 2018 Open Access This article is distributed under the terms of the Creative Commons Attribution 4.0 International License (http://creativecommons.org/licenses/by/4.0/), which permits unrestricted use, distribution, and reproduction in any medium, provided you give appropriate credit to the original author(s) and the source, provide a link to the Creative Commons license, and indicate if changes were made. The Creative Commons Public Domain Dedication waiver (http://creativecommons.org/publicdomain/zero/1.0/) applies to the data made available in this article, unless otherwise stated. 


\section{Background}

Three key behaviors which increase the risk of obesity in children are low levels of physical activity, excess time spent sedentary, and inadequate sleep [1-3]. While it is clear that each of these behaviors have independent effects on health, they also interact in ways that may not be apparent if studied individually [3, 4]. However, the majority of research has examined the effects of physical activity, sedentary behavior, and sleep in isolation [5]. This is flawed because time spent in one behavior will naturally depend on the composition of the rest of the day due to the finite nature of the 24-h window: if one component increases (e.g. sleep), then another component within the same 24-h block must decrease (e.g. sedentary time or physical activity or both) [4]. This means that 24-h movement data require different methods of analysis than standard multivariate techniques to take into account the co-dependence and proportional nature of compositional data $[6,7]$.

Despite the release of 24-h movement guidelines for preschool children in several countries [8,9], relatively few studies have collected 24-h movement data and even fewer have used appropriate compositional techniques to evaluate relationships with health [10-13]. Studies in older children and adolescents have demonstrated that each individual movement component (e.g. sleep, sedentary behavior) is independently related to BMI or fitness [11-13]. In the single study undertaken in preschool aged children, the overall composition of movement behaviors was related to BMI z-score, but not each individual component relative to the other components [10].

To date, all studies are cross-sectional in nature, which limits clarification of the benefits of replacing time in one behavior with another [3]. Only two studies appear to have used 24-h accelerometry to assess all components of movement patterns [11, 12]; the others have used accelerometry data to measure physical activity and sedentary time with questionnaires [10] or time diaries [13] to estimate sleep duration. However, it is unlikely that questionnaires are sensitive enough to detect the marked night-to-night variability in sleep duration that is revealed when 24-h accelerometry analyses are used in children [14]. No studies have investigated 24-h movement behaviors in infants and toddlers using compositional analyses, although a small body of work has examined adherence to new physical activity guidelines [15-17]. Similarly, none have used more accurate measures of adiposity than BMI to determine relationships with compositional time use, nor investigated other facets of body composition such as bone health [3].

Our recent Prevention of Overweight in Infancy (POI) study collected 24-h accelerometry data on a large sample of children for one week at 1, 2, 3.5 and 5 years of age, as well as accurate measures of body composition including bone health at 5 years of age. Therefore, the aims of this analysis were to i) describe 24-h movement behaviors from 1 to 5 years of age, ii) determine cross-sectional relationships between 24-h movement behaviors and body mass index (BMI) z-score, and iii) determine whether movement behaviors from 1 to 5 years of age predict body composition and bone health at 5 years.

\section{Methods}

Data from the POI randomised controlled trial were used. POI was an obesity prevention initiative investigating whether anticipatory guidance to parents about infant sleep, food, activity, and breastfeeding resulted in different growth patterns compared with usual care in children followed from before birth to 5 years of age. As the intervention did not produce evidence for differences in physical activity, sedentary behavior, or sleep [18-20], these analyses have used the entire cohort with adjustment for randomisation group.

As detailed information on the original trial is available in both the study protocol [21] and published findings [19, 20], only brief details are provided here. The original intervention was approved by the Lower South Regional Ethics Committee (LRS/12/08/063) and the follow-up study by the University of Otago Human Ethics Committee (12/ 274). Written informed consent was obtained from the parent/guardian of all child participants. All mothers who had booked into the single maternity hospital (> 97\% of all births) serving the population of Dunedin, New Zealand were invited to participate when in mid to late pregnancy (May 2009 to December 2010). A 58\% response rate yielded a final sample size of 802 primiparous (47.6\%) and multiparous (52.4\%) mothers. All anthropometric assessments and accelerometry analyses were performed by researchers blinded to group allocation.

\section{Measures obtained at baseline}

Demographic information obtained at baseline (late pregnancy) included maternal age, education, ethnicity, self-reported pre-pregnancy height and weight, and level of household deprivation [22]. Information on infant gestational age, sex and birth weight was obtained from hospital records.

\section{Measures obtained at 1, 2, 3.5 and 5 years of age}

Anthropometric measurements were obtained by trained research staff following standard protocols [23] at each age. Duplicate measures of weight (Tanita WB-100 MA/ WB-110 MA) and height (Harpenden stadiometer, Holtain Ltd., UK) were obtained with children wearing underwear/light clothing [24]. Quality control testing showed inter-rater reliability was excellent at each time point (>0.93). BMI z-scores were determined using the 
WHO growth standards [25], with overweight defined as a BMI $z$-score $\geq 85$ th but $<95$ th percentile, and obesity as $\mathrm{BMI} \geq 95$ th percentile.

Physical activity and sleep were assessed with children wearing Actical (Mini-Mitter, Bend, OR) accelerometers 24-h a day for up to 7 days at each age. The accelerometers were worn around the waist on elastic belts and initialized using $15 \mathrm{~s}$ epochs. Data were cleaned and scored using an automated script developed in MATLAB (MathWorks, Natick, MA, USA) that uses a count-scaled algorithm to estimate sleep onset and offset for overnight sleep, awakenings (wake after sleep onset, WASO) and daytime naps specific to each individual for each day $[26,27]$. Once these sleep data are effectively "removed" from the 24-h day, time spent sedentary and at various intensities of activity can be determined. Non-wear time was defined as at least 20 min of consecutive zeros during awake time data only [28], which provides acceptable estimates of physical activity $(r=0.70$ $0.90)$ in young children $[29,30]$. As Actical cutoffs for physical activity intensity categories do not exist that span this full age range (1-5 years), we defined sedentary time as 0 25 counts (per $15 \mathrm{~s}$ ), light physical activity (LPA) as 26-697 counts, and moderate-to-vigorous physical activity (MVPA) as $\geq 698$ counts [31], based on validation work in toddlers and preschoolers [32-34]. Data from our group shows that Actical accelerometers worn around the waist provide a valid measure of sleep duration in young children when compared against polysomnography (Meredith-Jones, unpublished). At 1 year of age, parents were asked whether the infant could walk alone (defined as "baby takes at least five steps independently in an upright position - there is no contact with a person or object"). All analyses at 1 year of age used this measure to adjust for whether or not the child was walking.

\section{Measures obtained at 5 years of age only}

Children underwent one total body DXA scan to assess body composition at 5 years of age only. DXA scans were performed and analysed by one experienced operator using a Lunar Prodigy scanner (GE Medical Systems Lunar, Madison WI); standard scanning procedures were used [35] and scans were analysed using the Lunar software package, version 16.0, with the pediatric option selected. Body composition variables assessed were percent body fat (fat mass as a percentage of body weight), bone mineral content (BMC, g) and bone mineral density $\left(\mathrm{BMD}, \mathrm{g} / \mathrm{cm}^{2}\right)$. Both bone measures were total body less head (TBLH) as recommended in children [36].

\section{Statistical analyses}

\section{Normalising to $24 \mathrm{~h}$}

In order to keep each overnight sleep as a solid block of data, each 24-h 'day' was determined from the time the child woke up on day 1 , to the time they woke up on day 2 (and so on). However, this means that each 'day' does not add up to 1440 min (as it might if midnight to midnight periods were used for example). A day was thus considered valid if the participant had between 20 and $28 \mathrm{~h}$ of data to allow for changing wake times across different days. Currently, there is no consensus on what represents a valid data when examining 24-h data. While others [11] have used $16 \mathrm{~h}$ a day (for at least 3 days), we believe this had the potential to include a lot of non-wear time given $16 \mathrm{~h}$ represents just $67 \%$ of a $24-\mathrm{h}$ day. Our use of at least $20 \mathrm{~h}$ ( $83 \%$ of a 24 -h day) was chosen to provide greater confidence in minimising non-wear time. In our $24 \mathrm{~h}$ data, non-wear time was less than $5 \%$. This meant that the sleep and activity components had to be normalised to $24 \mathrm{~h}$. Before this, the components during awake day time (naps; sedentary, light, moderate and vigorous activity) were normalised for all awake day time (including non-wear time), on the assumption that non-wear time (such as device removal for bathing) did not occur overnight when children were asleep. Otherwise, simply ignoring non-wear time would essentially allocate, incorrectly, some of the non-wear time to night-time sleep and time awake after sleep onset. Normalisation was undertaken by multiplying the proportion of time spent in each component for the 'day' by $1440 \mathrm{~min}(24 \mathrm{~h})$.

\section{Weighting for weekend and weekday data}

Participants needed to have at least three valid days of data to be included, which could have included a mix of weekdays and weekend days. Weekdays were weighted so that all weekdays together had a weight of $5 / 7$, and weekend days were similarly weighted so that all weekend days had a weight of $2 / 7$, making the data representative of a full week.

\section{Descriptive statistics}

Both arithmetic and compositional means were calculated for each component. Compositional means are found by taking the geometric means for each of the components and dividing by the sum of all component geometric means, then normalising to $24 \mathrm{~h}$ by multiplying by 1440 min. Standard deviations of compositional means are not appropriate, instead the variances of the $\log$ ratios between the components were calculated. This is a measure of how proportional two components are - values closer to zero have a higher degree of proportionality.

\section{Isometric log-ratio coordinates}

Due to the closed nature of the 24-h movement data, perfect multicollinearity exists and therefore traditional multivariable statistical methods are inappropriate. Recent studies into the compositional nature of 24-h movement and BMI outcomes have recommended the use of isometric log-ratio (ilr) coordinates, where the 
transformation of the data is well-described $[7,12]$. Because log-ratios become problematic with zeros, time spent in day time naps and wake after sleep onset (time spent awake in the night between sleep onset and sleep offset) were reallocated to time spent asleep and sedentary, respectively. A set of ilr coordinates was calculated for each component of the day (i.e. a set for each of sleep, sedentary, light and MVPA). As there were four components in the day, there were four sets of ilr coordinates, with three coordinates in each set.

\section{Associations between 24-h movement patterns and body composition and bone health}

Separate regression models were used for each set of ilr coordinates (e.g. the sleep set). The regression coefficient of the first ilr coordinate was used to describe the relationship between the time component (e.g. sleep) and the outcome (e.g. BMI z-score) because the first ilr coordinate is proportional to the log ratio of the component time and the geometric mean of the remaining component times. This regression coefficient can be back-transformed to give a meaningful estimate of the strength of the association. Estimates are described in terms of "time reallocation". This terminology is used because the predictor variable is a function of the ratio between component time (the numerator) and remaining component times (the denominator). Therefore a "unit change" in the predictor variable is a measure of how much time from the particular component is reallocated to time in all other components (i.e. from the numerator to the denominator or vice versa). We used the methods as described by Dumuid et al. [12] to give the estimated difference in the outcome variable (e.g. BMI z-score) associated with a $10 \%$ difference in time allocation to a specific component (e.g. sleep). As the relationship is non-linear, an estimated difference for both a $10 \%$ greater and a $10 \%$ lower time allocation was calculated, along with $95 \%$ confidence intervals for this estimate. A $10 \%$ difference in time allocation was chosen in preference to the use of a set number of minutes (e.g. 15,30 etc) to allow consistency in the size of the effect examined; $15 \mathrm{~min}$ of MVPA for example represents a much larger proportion of total daily MVPA than 15 min of sleep does for total daily sleep.

Linear regression models were used to determine cross-sectional, longitudinal and prospective associations all adjusted for randomised group, sex and demographic variables (and walking status for BMI z-score analyses at 1 year of age). BMI $z$-score at all ages, and percent body fat, and total body less head BMD $\left(\mathrm{g} / \mathrm{m}^{2}\right)$ and BMC (g) at 5 years of age were the outcome variables. The longitudinal associations with BMI z-score were adjusted for concurrent BMI z-score. The prospective bone analyses were further adjusted for BMI $\mathrm{z}$-score given the known differences in bone relating to body size in children. Residuals were plotted and visually assessed for normality and homogeneity of variance. Missing data was excluded list-wise. All data were analysed using Stata 15.1 (StataCorp, Texas).

\section{Results}

Table 1 presents the characteristics of the study population at baseline (birth) from the original POI study, and those with available data for analyses. Overall, mothers were predominantly New Zealand European (84.1\%), and two-thirds (67.2\%) had a University education. Approximately half the group were having their first child (48.3\%), and fewer children came from homes with higher levels of neighbourhood deprivation than is seen nationally (i.e. $16.5 \%$ compared to the expected $30 \%$ ).

Table 2 presents the characteristics of participants at each point who had valid accelerometry data. Children who provided at least one set of valid accelerometry data $(n=380)$ compared to children providing no accelerometry data at any age $(n=422)$ had mothers who were older at birth (32.5 years vs 30.7 years, $p<0.001)$, and more highly educated $(66.1 \%$ had a tertiary education compared to $56.5 \%, p=0.019)$, but were not different according to ethnicity ( $p=0.722)$ pre-pregnancy BMI ( $p=$ $0.090)$, household deprivation $(p=0.070)$, infant $\operatorname{sex}(p=$ $0.970)$ or birthweight $(p=0.162)$.

Figure 1 demonstrates the differential time allocation from 1 to 5 years of age. At 1 year of age, infants spent nearly $50 \%$ of their 24-h day asleep (arithmetic mean of $699 \mathrm{~min}$ or $11.7 \mathrm{~h}$ including naps), almost $9 \mathrm{~h}$ being sedentary, about $3 \mathrm{~h}$ in light intensity activity, and virtually no time in MVPA. Marked changes occur between 1 and 2 years of age, with a substantial increase in light intensity activity in particular ( $100 \mathrm{~min}$ more per day on average). Over these four years, gradual increases in MVPA are observed, with children spending $33 \mathrm{~min}$ on average each day, $1.9 \%$ of the $24-\mathrm{h}$ period, by 5 years of age. Additional file 1: Table S1 shows the variances of the $\log$ ratios, which indicate that time spent asleep, sedentary and in LPA had high proportionality, especially after 12 months of age.

No cross-sectional relationships were observed between compositional time use and BMI z-score at 1,2 or 5 years of age (Table 3 ). Similarly, compositional time use was not related to BMI z-score when measured longitudinally (Table 4). However, at 3.5 years of age, spending $10 \%$ more time asleep (65 additional minutes) relative to other behaviors was associated with a lower BMI z-score (estimated difference, - 0.248: 95\% CI -0.418 to -0.080 ) whereas spending $10 \%$ more of the day in LPA $(29 \mathrm{~min})$ or being sedentary $(47 \mathrm{~min})$ was 
Table 1 Demographic characteristics of the study population at baseline

\begin{tabular}{|c|c|c|c|c|}
\hline & & All POI participants & $\begin{array}{l}\text { Those with at least one } \\
\text { set of data } \mathrm{n}(\%)\end{array}$ & $\begin{array}{l}\text { Those with at least one set of } \\
\text { data }^{a} \text { and DXA at } 5 \text { years } n(\%)\end{array}$ \\
\hline$n$ & & 802 & 380 & 257 \\
\hline \multicolumn{5}{|l|}{ Maternal variables } \\
\hline Age at child's birth (y) & Mean (SD) & $31.6(5.2)$ & $32.5(4.7)$ & $33.2(4.4)$ \\
\hline \multirow[t]{4}{*}{ Ethnicity n (\%) } & New Zealand European & $682(85.1)$ & $299(78.7)$ & $216(84.1)$ \\
\hline & Māori & $46(5.7)$ & $36(9.5)$ & $18(7.0 \%)$ \\
\hline & Other & $73(9.1)$ & $45(11.8)$ & $23(9.0)$ \\
\hline & Missing & 1 & 2 & 0 \\
\hline \multirow[t]{2}{*}{ Parity n (\%) } & Primiparous & $382(47.6)$ & $187(49.2)$ & $124(48.3)$ \\
\hline & Multiparous & $420(52.4)$ & $193(50.8)$ & $133(51.8)$ \\
\hline \multirow[t]{4}{*}{ Education $^{\mathrm{b}} \mathrm{n}(\%)$} & School only & $193(24.1)$ & 78 (20.6) & $48(18.8)$ \\
\hline & Post-secondary & $116(14.6)$ & $50(13.2)$ & $36(14.1)$ \\
\hline & University degree or higher & $485(61.1)$ & $250(66.1)$ & $172(67.2)$ \\
\hline & Missing & 8 & 2 & 1 \\
\hline Pre-pregnancy BMI $\left(\mathrm{kg} / \mathrm{m}^{2}\right)$ & Mean (SD) & $25.1(5.0)$ & $25.4(5.1)$ & $25.3(5.2)$ \\
\hline \multicolumn{5}{|l|}{ Household or infant variables } \\
\hline \multirow[t]{4}{*}{ Household deprivation $^{c}$} & 1-3 (Low) & $276(34.8)$ & $140(37.0)$ & $102(40.0)$ \\
\hline & $4-7$ & $350(43.6)$ & $171(45.2)$ & $111(43.5)$ \\
\hline & 8-10 (High) & $168(21.2)$ & $67(17.7)$ & $42(16.5)$ \\
\hline & Missing & 8 & 2 & 2 \\
\hline \multirow[t]{2}{*}{ Infant sex } & Male & $391(48.8)$ & $195(51.3)$ & $125(48.6)$ \\
\hline & Female & $411(51.2)$ & $185(48.7)$ & $132(51.4)$ \\
\hline Infant birth weight (kg) & Mean (SD) & $3551(480)$ & $3576(492)$ & $3581(496)$ \\
\hline
\end{tabular}

Data presented as $\mathrm{n}(\%)$ unless otherwise indicated

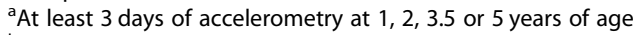

becondary schooling in New Zealand is from year 9 to year 13 inclusive, 'post-secondary qualifications' refer to all tertiary qualifications that are not University based

'Uses the New Zealand Index of Deprivation 2013 which combines nine variables from the 2013 census relating to communication (no access to the internet at home), income (receiving a means tested benefit or living below income thresholds), unemployment, qualifications, home ownership, single parent families, living space, and transport access. A deprivation score is provided for each meshblock which is a geographical unit defined by Statistics New Zealand containing about 60-110 people. The score reflects the extent of material and social deprivation and is used to construct deciles from 1 (least deprived) to 10 (most deprived)

associated with higher BMI z-scores $(0.122,0.010$ to 0.23 and $0.081,0.015$ to 0.147 respectively, Table 3$)$. Tables 5 (lean mass) and 6 (percent fat mass) demonstrate that these differences in BMI reflect differences in lean mass rather than fat mass.

In terms of bone health, higher levels of MVPA were consistently associated with higher TBLH BMD (Table 7) and TBLH BMC (Table 8). A 10\% difference in MVPA at 2 to 5 years of age ( 0.6 to $3 \mathrm{~min}$ a day) produced statistically significant, albeit small, differences in TBLH BMD of 0.001 to $0.002 \mathrm{~g} / \mathrm{cm}^{2}$ at the different ages. Very similar small effect sizes were observed in regards to MVPA and TBLH BMC (Table 8). Although a 10\% increase in MVPA was associated with statistically significant differences in TBLH BMC, these values of 2.0 to $3.2 \mathrm{~g}$ would be considered negligible for practical purposes. No consistent relationships between other components of compositional time use and bone health were apparent (Tables 7 and 8).

\section{Discussion}

Our data demonstrate that considerable changes in compositional time use occur across the 24-h day over the preschool years. In late infancy, children are spending just under $12 \mathrm{~h}$ asleep on average, about $3 \mathrm{~h}$ in activity of light intensity, and the majority of awake time in sedentary pastimes. By 5 years of age, substantial increases in light-intensity activity occur, as do increases in MVPA, with corresponding reductions in time spent asleep, particularly as naps during the day. However, compositional time use showed little relationship to body composition, whether assessed cross-sectionally, prospectively, or longitudinally. Spending more time asleep or less time in LPA or sedentary activity were associated cross-sectionally with lower BMI z-scores at 3.5 years of age, but significant relationships were not observed at any other age or longitudinally. Although higher time spent in MVPA was positively associated with bone health, the actual 
Table 2 Characteristics of the group at each time point including allocation of time use

\begin{tabular}{|c|c|c|c|c|c|}
\hline \multirow[t]{2}{*}{ Variable } & \multirow[t]{2}{*}{ Category } & \multicolumn{4}{|l|}{ Age (years) } \\
\hline & & 1 & 2 & 3.5 & 5 \\
\hline$n$ & & 355 & 213 & 231 & 248 \\
\hline Female n (\%) & & $172(48.5)$ & $93(43.7)$ & $107(46.3)$ & $122(49.2)$ \\
\hline Age (months) & & $12.2(0.3)$ & $24.1(0.2)$ & $41.9(0.5)$ & $59.7(0.4)$ \\
\hline Height (cm) & & $75.6(2.8)$ & $86.2(3.1)$ & $99.1(3.8)$ & $109.9(4.4)$ \\
\hline Weight (kg) & & $9.8(1.1)$ & $12.5(1.4)$ & $16.0(1.8)$ & $19.4(2.4)$ \\
\hline $\mathrm{BMI}\left(\mathrm{kg} / \mathrm{m}^{2}\right)$ & & $17.2(1.3)$ & $16.8(1.3)$ & $16.3(1.2)$ & $16.0(1.2)$ \\
\hline BMI z-score & & $0.39(0.83)$ & $0.70(0.89)$ & $0.61(0.83)$ & $0.47(0.81)$ \\
\hline \multirow[t]{3}{*}{ Weight status ${ }^{a} \mathrm{n}(\%)$} & Normal weight & $281(79.2)$ & $140(65.7)$ & $158(68.4)$ & $190(76.6)$ \\
\hline & Overweight & $52(14.7)$ & $43(20.2)$ & 45 (19.5) & $40(16.1)$ \\
\hline & Obese & $22(6.2)$ & $30(14.1)$ & $28(12.1)$ & $18(7.3)$ \\
\hline Sleep components ${ }^{\mathrm{b}}$ & Night-time sleep & $578(55)$ & $594(60)$ & $626(44)$ & $632(39)$ \\
\hline \multirow[t]{2}{*}{ (minutes) } & Wake after sleep onset & $29(27)$ & $20(22)$ & $15(16)$ & $12(14)$ \\
\hline & Day time naps & $121(43)$ & $89(42)$ & $23(26)$ & $-c$ \\
\hline Activity components ${ }^{\mathrm{b}}$ & Sedentary & $524(58)$ & $443(61)$ & $462(54)$ & $476(58)$ \\
\hline \multirow[t]{2}{*}{ (minutes) } & LPA & $184(64)$ & $284(56)$ & $292(54)$ & $287(54)$ \\
\hline & MVPA & $4(6)$ & $10(8)$ & $22(14)$ & $33(17)$ \\
\hline
\end{tabular}

Data presented as mean (SD) except for weight status which is $\mathrm{n}(\%)$ and only includes those with valid accelerometry data LPA is light physical activity, MVPA is moderate-to-vigorous physical activity

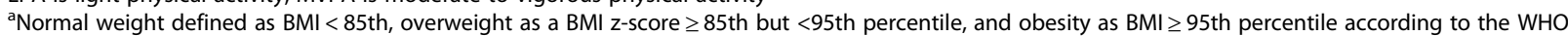
growth standards [25]

${ }^{\mathrm{b}}$ Data presented as arithmetic mean (SD) which is the average number of minutes spent in each category after individualised normalisation to $24 \mathrm{~h}$ ${ }^{\mathrm{c} C o u l d}$ not be calculated as no children napped at this age

differences observed were very small and unlikely to be of clinical significance. In combination, although observational, these findings would provide little support for compositional time use being associated with body composition in young children.

The degree to which sedentary behavior, physical activity, and/or sleep influence body composition and bone health in young preschool-aged children is uncertain. A series of recent comprehensive systematic reviews have highlighted that while objectively measured sedentary behavior appears to have little association with adiposity or bone health [37], the majority of studies support an adverse effect of shortened sleep on weight status [38]. In terms of physical activity, findings are more mixed. Although time spent in MVPA has frequently been associated with reduced adiposity and improved bone health in observational research, meta-analyses of the few intervention studies did not show any difference in BMI according to activity levels [39]. Moreover, time in light and moderate intensity activity was not consistently associated with favourable health outcomes [39]. As highlighted in these reviews, the risk of bias is high for much of this evidence, leading to overall gradings of low or very low for the quality of the evidence, according to GRADE frameworks [40]. These mixed findings help illustrate the importance of compositional analyses, particularly when others have shown that omitting individual behaviors (such as sleep) from models can substantially affect the interpretation of the relationships between the remaining behaviors and BMI, including changing the direction of the relationships [12].

It is difficult to compare our findings with the literature given the scarcity of compositional time-use studies, particularly in preschool children. Studies in older children and adolescents are all cross-sectional and have generally provided estimates for the effect on BMI $\mathrm{z}$-score of reallocating time in one activity to another activity. For example, in one study in 10-year old children, the predicted difference in BMI z-score if $15 \mathrm{~min}$ of sleep is replaced with $15 \mathrm{~min}$ of MVPA per day is -0.48 . Interestingly, the effect is not linear, that is, doing 15 min per day less MVPA to gain 15 min of sleep results in a predicted difference in BMI z-score of 0.88 , almost twice that of the reverse direction [11]. Others have also demonstrated seemingly substantial benefits to BMI of substituting sedentary behavior for MVPA; replacing one hour of sedentary time with the same amount of MVPA was associated with a difference in BMI of up to $1.4 \mathrm{~kg} /$ $\mathrm{m}^{2}$ across a wide age range (5-19 years) [13]. These differences in BMI are substantial, and upon initial inspection provide significant support for an important impact of MVPA on BMI in children. However, these studies hypothesize a somewhat unrealistic substitution scenario where time spent in one behavior would be entirely 


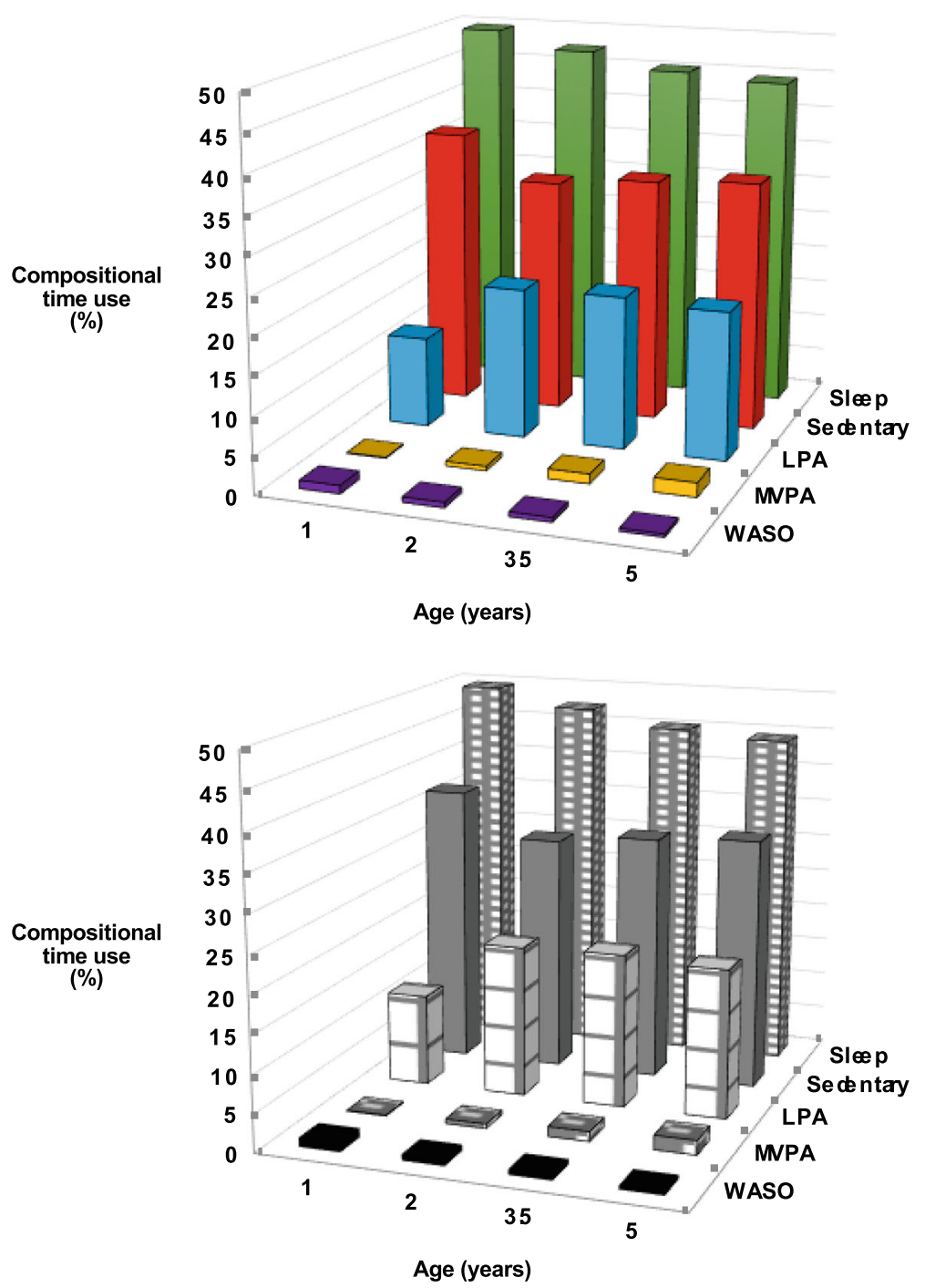

Fig. 1 Compositional time use (\% of 24-h day) at different ages. Data presented as compositional means - the geometric mean that has been re-normalised to $24 \mathrm{~h}$ (1440 $\mathrm{min}$ ) for all component geometric means. Data presented as minutes (\% of $1440 \mathrm{~min}$ ) after normalisation to $24 \mathrm{~h}$ for each participant. Night-time sleep and naps have been combined. LPA is light physical activity, MVPA is moderate-to-vigorous physical activity, WASO is wake after sleep onset

reallocated to one other behavior with no effect on the remaining behaviors, ignoring the co-dependent nature of the behaviors. The biggest differences are consistently seen when substituting a number of minutes for MVPA. This is because MVPA, while possibly having an influence on body composition, is the smallest component in the day, where a $15 \mathrm{~min}$ reallocation is, for example, $57 \%$ of mean MVPA time but only $3 \%$ of mean sleep time [11]. Therefore it isn't the small, insignificant reallocation of sleep that contributes to the estimated difference in BMI z-score but rather the substantial reallocation to MVPA. However, presenting estimates in this way can give a misleading view of what behaviors should be encouraged and discouraged. We have attempted to present associations between time-use and body composition in a more transparent and realistic way, as described by Dumuid and colleagues [41], by reporting on the reallocation of a proportion of the component (not a set number of minutes) from one activity to all others [41].

The single existing compositional time-use study in preschool aged children reported that overall time use was associated with BMI z-score in 3-4year old children, but that significant effects were not apparent for any of the individual components of time use in cross-sectional analyses [10]. Although the present study demonstrated that sleep was inversely associated, and 
Table 3 Cross-sectional relationships between sleep and activity components and BMI z-score at each age

\begin{tabular}{|c|c|c|c|c|c|c|c|}
\hline Age (years) & $\begin{array}{l}\text { Mean } \\
\text { (SD) BMl } \\
\text { z-score }\end{array}$ & Component & $\begin{array}{l}\text { Regression } \\
\text { coefficient }^{\mathrm{b}} \\
\text { (SE) }\end{array}$ & $p$-value & $\begin{array}{l}10 \% \text { relative } \\
\text { difference in } \\
\text { minutes }\end{array}$ & $\begin{array}{l}\text { Estimated difference }(95 \% \mathrm{Cl}) \text { in } \\
\text { BMI z-score with a } 10 \% \text { increase } \\
\text { in component }\end{array}$ & $\begin{array}{l}\text { Estimated difference }(95 \% \mathrm{Cl}) \text { in } \\
\text { BMl z-score with a } 10 \% \text { decrease } \\
\text { in component }\end{array}$ \\
\hline \multirow[t]{4}{*}{$1(n=346)$} & $0.39(0.83)$ & Sleep $^{c}$ & $0.00(0.30)$ & 0.989 & 71 & $-0.001(-0.099,0.097)$ & $0.001(-0.098,0.099)$ \\
\hline & & Sedentary ${ }^{d}$ & $0.02(0.30)$ & 0.934 & 56 & $-0.008(-0.087,0.072)$ & $0.008(-0.074,0.090)$ \\
\hline & & LPA & $0.03(0.14)$ & 0.816 & 18 & $0.010(-0.013,0.033)$ & $-0.011(-0.036,0.014)$ \\
\hline & & MVPA & $-0.06(0.04)$ & 0.130 & 0.1 & $-0.004(-0.010,0.002)$ & $0.004(-0.003,0.012)$ \\
\hline \multirow[t]{4}{*}{$2(n=213)$} & $0.70(0.89)$ & Sleep & $0.45(0.40)$ & 0.265 & 69 & $0.074(-0.056,0.204)$ & $-0.075(-0.205,0.056)$ \\
\hline & & Sedentary & $-0.28(0.37)$ & 0.448 & 46 & $-0.035(-0.124,0.055)$ & $0.037(-0.058,0.131)$ \\
\hline & & LPA & $-0.08(0.33)$ & 0.804 & 28 & $-0.009(-0.076,0.059)$ & $0.009(-0.064,0.082)$ \\
\hline & & MVPA & $-0.09(0.08)$ & 0.285 & 0.6 & $-0.007(-0.020,0.006)$ & $0.008(-0.006,0.022)$ \\
\hline \multirow[t]{4}{*}{$3.5(n=231)$} & $0.61(0.83)$ & Sleep & $-1.57(0.55)$ & 0.005 & 65 & $-0.248(-0.418,-0.080)$ & $0.252(0.079,0.425)$ \\
\hline & & Sedentary & $0.97(0.45)$ & 0.034 & 47 & $0.122(0.010,0.235)$ & $-0.129(-0.247,-0.011)$ \\
\hline & & LPA & $0.77(0.32)$ & 0.017 & 29 & $0.081(0.015,0.147)$ & $-0.087(-0.158,-0.016)$ \\
\hline & & MVPA & $-0.17(0.13)$ & 0.182 & 2 & $-0.014(-0.036,0.007)$ & $0.016(-0.007,0.039)$ \\
\hline \multirow[t]{4}{*}{$5(n=248)$} & $0.44(0.84)$ & Sleep & $-0.89(0.54)$ & 0.100 & 64 & $-0.137(-0.300,0.026)$ & $0.140(-0.026,0.306)$ \\
\hline & & Sedentary & $0.25(0.41)$ & 0.534 & 49 & $0.032(-0.070,0.135)$ & $-0.034(-0.141,0.073)$ \\
\hline & & LPA & $0.55(0.30)$ & 0.072 & 28 & $0.057(-0.005,0.118)$ & $-0.061(-0.128,0.005)$ \\
\hline & & MVPA & $0.09(0.13)$ & 0.481 & 3 & $0.008(-0.014,0.029)$ & $-0.008(-0.032,0.015)$ \\
\hline
\end{tabular}

LPA is light physical activity, MVPA is moderate-to-vigorous physical activity

${ }^{a}$ Adjusted for randomised group, sex, primiparous, maternal education, household deprivation, ethnicity and maternal BMI. Analyses at 1 year of age further adjusted for whether the child was walking unaided or not walking

${ }^{b}$ Coefficient for the first isometric log ratio (ilr) co-ordinate, which is proportional to the log ratio of the behavior and the geometric mean of the remaining behaviors; adjusted for remaining ilr co-ordinates

${ }^{c}$ All sleep estimates include naps where relevant

${ }^{\mathrm{d}}$ All sedentary estimates include wake after sleep onset

Bolded values indicate $P<0.05$

Table 4 Predictive relationships between sleep and activity components at 1, 2 and 3.5 years of age, and BMI z-score at 5 years ${ }^{a}$

\begin{tabular}{|c|c|c|c|c|c|c|c|}
\hline Age (years) & $\begin{array}{l}\text { Mean BMl z- } \\
\text { score (SD) at } 5 \\
\text { years of age }\end{array}$ & Component & $\begin{array}{l}\text { Regression } \\
\text { coefficient }{ }^{\mathrm{b}} \\
\text { (SE) }\end{array}$ & $p$-value & $\begin{array}{l}10 \% \text { relative } \\
\text { difference in } \\
\text { minutes }\end{array}$ & $\begin{array}{l}\text { Estimated difference }(95 \% \mathrm{Cl}) \text { in } \\
\text { BMl z-score with a } 10 \% \text { increase } \\
\text { in component }\end{array}$ & $\begin{array}{l}\text { Estimated difference }(95 \% \mathrm{Cl}) \text { in } \\
\text { BMI z-score with a } 10 \% \text { decrease } \\
\text { in component }\end{array}$ \\
\hline \multirow[t]{4}{*}{$1(n=292)$} & \multirow[t]{4}{*}{$0.43(0.83)$} & Sleep ${ }^{c}$ & $-0.24(0.24)$ & 0.336 & 71 & $-0.038(-0.119,0.043)$ & $0.038(-0.043,0.119)$ \\
\hline & & Sedentary ${ }^{d}$ & $0.28(0.25)$ & 0.266 & 56 & $0.024(-0.042,0.089)$ & $-0.024(-0.092,0.043)$ \\
\hline & & LPA & $-0.08(0.12)$ & 0.510 & 18 & $0.002(-0.017,0.021)$ & $-0.002(-0.023,0.019)$ \\
\hline & & MVPA & $0.03(0.03)$ & 0.312 & 0.1 & $0.003(-0.003,0.008)$ & $-0.003(-0.009,0.003)$ \\
\hline \multirow[t]{4}{*}{$2(n=190)$} & \multirow[t]{4}{*}{$0.43(0.89)$} & Sleep & $-0.43(0.28)$ & 0.126 & 69 & $-0.071(-0.162,0.020)$ & $0.072(-0.020,0.164)$ \\
\hline & & Sedentary & $0.45(0.26)$ & 0.082 & 46 & $0.056(-0.007,0.120)$ & $-0.059(-0.126,0.007)$ \\
\hline & & LPA & $-0.05(0.24)$ & 0.817 & 28 & $-0.006(-0.054,0.042)$ & $0.006(-0.046,0.058)$ \\
\hline & & MVPA & $0.03(0.06)$ & 0.559 & 0.6 & $0.003(-0.007,0.012)$ & $-0.003(-0.013,0.007)$ \\
\hline \multirow[t]{4}{*}{$3.5(n=212)$} & \multirow[t]{4}{*}{$0.44(0.84)$} & Sleep & $-0.08(0.30)$ & 0.784 & 65 & $-0.013(-0.107,0.081)$ & $0.013(-0.082,0.108)$ \\
\hline & & Sedentary & $-0.24(0.25)$ & 0.326 & 47 & $-0.031(-0.091,0.030)$ & $0.032(-0.032,0.096)$ \\
\hline & & LPA & $0.23(0.17)$ & 0.183 & 29 & $0.024(-0.011,0.060)$ & $-0.026(-0.065,0.012)$ \\
\hline & & MVPA & $0.09(0.07)$ & 0.182 & 2 & $0.008(-0.004,0.019)$ & $-0.009(-0.021,0.004)$ \\
\hline
\end{tabular}

LPA is light physical activity, MVPA is moderate-to-vigorous physical activity

${ }^{a}$ Adjusted for concurrent BMI Z-score, randomised group, sex, primiparous, maternal education, household deprivation, ethnicity and maternal BMI. Analyses at 1 year of age further adjusted for whether the child was walking unaided or not walking

${ }^{b}$ Coefficient for the first isometric log ratio (ilr) co-ordinate, which is proportional to the log ratio of the behavior and the geometric mean of the remaining behaviors; adjusted for remaining ilr co-ordinates

${ }^{c}$ All sleep estimates include naps where relevant

${ }^{\mathrm{d}}$ All sedentary estimates include wake after sleep onset 
Table 5 Predictive and concurrent relationships between sleep and activity components at 1, 2, 3.5 and 5 years of age and fat-free mass index at 5 years ${ }^{a}$

\begin{tabular}{|c|c|c|c|c|c|c|c|}
\hline Age (years) & $\begin{array}{l}\text { Mean fat free mass } \\
\text { index }(S D) \text { at } 5 \text { years } \\
\text { of age, } \mathrm{kg} / \mathrm{m}^{2}\end{array}$ & Component & $\begin{array}{l}\text { Regression } \\
\text { coefficient }^{\mathrm{b}} \\
\text { (SE) }\end{array}$ & $p$-value & $\begin{array}{l}10 \% \\
\text { difference } \\
\text { in minutes }\end{array}$ & $\begin{array}{l}\text { Estimated difference }(95 \% \mathrm{Cl}) \\
\text { in } \% \text { body fat with a } 10 \% \\
\text { increase in component }\end{array}$ & $\begin{array}{l}\text { Estimated difference }(95 \% \mathrm{Cl}) \\
\text { in } \% \text { body fat with a } 10 \% \\
\text { decrease in component }\end{array}$ \\
\hline \multirow[t]{4}{*}{$1(n=229)$} & $12.7(0.8)$ & Sleep $^{c}$ & $-0.46(0.32)$ & 0.144 & 71 & $-0.079(-0.184,0.026)$ & $0.079(-0.027,0.185)$ \\
\hline & & Sedentary ${ }^{d}$ & $0.27(0.32)$ & 0.393 & 56 & $0.038(-0.049,0.124)$ & $-0.039(-0.129,0.050)$ \\
\hline & & LPA & $0.16(0.15)$ & 0.278 & 18 & $0.015(-0.012,0.043)$ & $-0.017(-0.046,0.013)$ \\
\hline & & MVPA & $0.03(0.04)$ & 0.488 & 0.1 & $0.003(-0.005,0.010)$ & $-0.003(-0.011,0.005)$ \\
\hline \multirow[t]{4}{*}{$2(n=158)$} & $12.8(0.8)$ & Sleep & $-0.32(0.35)$ & 0.365 & 69 & $-0.053(-0.166,0.061)$ & $0.053(-0.061,0.168)$ \\
\hline & & Sedentary & $0.60(0.32)$ & 0.065 & 46 & $0.075(-0.004,0.154)$ & $-0.079(-0.162,0.004)$ \\
\hline & & LPA & $-0.37(0.32)$ & 0.244 & 28 & $-0.039(-0.104,0.026)$ & $0.042(-0.028,0.112)$ \\
\hline & & MVPA & $0.09(0.08)$ & 0.234 & 0.6 & $0.008(-0.005,0.020)$ & $-0.008(-0.022,0.005)$ \\
\hline \multirow[t]{4}{*}{$3.5(n=178)$} & $12.8(0.8)$ & Sleep & $-2.19(0.53)$ & $<0.001$ & 65 & $-0.346(-0.509,-0.183)$ & $0.352(0.186,0.518)$ \\
\hline & & Sedentary & $0.87(0.43)$ & 0.046 & 47 & $0.110(0.003,0.217)$ & $-0.116(-0.229,-0.003)$ \\
\hline & & LPA & $1.26(0.31)$ & $<0.001$ & 29 & $0.132(0.070,0.195)$ & $-0.143(-0.210,-0.075)$ \\
\hline & & MVPA & $0.06(0.12)$ & 0.641 & 2 & $0.005(-0.015,0.024)$ & $-0.005(-0.027,0.017)$ \\
\hline \multirow[t]{4}{*}{$5(n=213)$} & $12.7(0.8)$ & Sleep & $-0.82(0.55)$ & 0.134 & 64 & $-0.127(-0.292,0.039)$ & $0.129(-0.039,0.298)$ \\
\hline & & Sedentary & $0.17(0.41)$ & 0.673 & 49 & $0.022(-0.080,0.124)$ & $-0.023(-0.131,0.084)$ \\
\hline & & LPA & $0.48(0.30)$ & 0.116 & 28 & $0.050(-0.012,0.111)$ & $-0.054(-0.120,0.013)$ \\
\hline & & MVPA & $0.17(0.13)$ & 0.170 & 3 & $0.015(-0.006,0.035)$ & $-0.016(-0.039,0.007)$ \\
\hline
\end{tabular}

LPA is light physical activity, MVPA is moderate-to-vigorous physical activity

${ }^{a}$ Adjusted for randomised group, sex, primiparous, maternal education, household deprivation, ethnicity and maternal BMI. Analyses at 1 year of age further adjusted for whether the child was walking unaided or not walking

${ }^{b}$ Coefficient for the first isometric log ratio (ilr) co-ordinate, which is proportional to the log ratio of the behavior and the geometric mean of the remaining behaviors; adjusted for remaining ilr co-ordinates

cAll sleep estimates include naps where relevant

${ }^{\mathrm{d}}$ All sedentary estimates include wake after sleep onset

Bolded values indicate $P<0.05$

sedentary and LPA positively associated with BMI, this was not a consistent finding. Our data clearly show that significant relationships between time use and BMI were only observed at one of the four cross-sectional time points (3.5 years of age). Importantly, compositional time use did not predict BMI at any of the longitudinal time points examined. Discrepancy in findings may be due to the use of questionnaires [10] versus actigraphy (current study) to assess sleep, and/or a result of different cutpoints to denote intensity of activity between studies. Use of different processing criteria is a well-known limitation of accelerometry analyses which limits direct comparison between studies [42]. Regardless of these issues, our analyses clearly demonstrate that although time use might be related to BMI cross-sectionally, no such relationships are apparent in longitudinal analyses, at least in this young cohort.

Our findings also indicate that variation in time use has no relationship with percentage body fat at this young age. We cannot compare our findings with the literature as no studies appear to have examined compositional time use over the full 24 -h period to evaluate relationships with percent fat in children [3]. Replacing sedentary time with MVPA has been associated with lower adiposity in the few existing studies in children that have used isotemporal analyses to determine how reallocation of time use influences adiposity [43, 44]. However, none of the studies included had measures of sleep and thus 24-h time use. Work from our group demonstrates that correctly accounting for sleep is crucial for obtaining accurate measures of sedentary time; estimates of sedentary time across a 24-h day ranged from 556 to 1145 min depending on the sleep scoring method utilised [26].

Engaging in sufficient moderate-to-vigorous physical activity is important for bone development [37], even in young children [45], and limited work suggests that sufficient sleep might also be important for bone [46]. However, no studies have examined combinations of physical activity, sedentary behavior, and sleep in relation to BMC or BMD in children [3]. Although our study consistently demonstrated a protective effect of MVPA at 2, 3.5 and 5 years of age on bone outcomes at 5 years of age, these differences were small, and unlikely to be clinically relevant as they fell within the expected error for repeat scans on children $\left(0.004 \mathrm{~g} / \mathrm{cm}^{2}\right.$ or $\left.0.6 \% \mathrm{CV}\right)$. By contrast, our findings regarding the impact of sleep and sedentary findings were much less consistent and thus should be interpreted with caution. Time spent asleep was not related to bone health, with a single 
Table 6 Predictive and concurrent relationships between sleep and activity components at 1, 2, 3.5 and 5 years of age and percent fat at 5 years ${ }^{\mathrm{a}}$

\begin{tabular}{|c|c|c|c|c|c|c|c|}
\hline Age (years) & $\begin{array}{l}\text { Mean \% body } \\
\text { fat (SD) at } 5 \\
\text { years of age }\end{array}$ & Component & $\begin{array}{l}\text { Regression } \\
\text { coefficient }^{\mathrm{b}} \\
\text { (SE) }\end{array}$ & $p$-value & $\begin{array}{l}10 \% \\
\text { difference } \\
\text { in minutes }\end{array}$ & $\begin{array}{l}\text { Estimated difference }(95 \% \mathrm{Cl}) \text { in } \\
\% \text { body fat with a } 10 \% \text { increase } \\
\text { in component }\end{array}$ & $\begin{array}{l}\text { Estimated difference }(95 \% \mathrm{Cl}) \text { in } \\
\% \text { body fat with a } 10 \% \text { decrease } \\
\text { in component }\end{array}$ \\
\hline \multirow[t]{4}{*}{$1(n=232)$} & $15.6(4.8)$ & Sleep $^{c}$ & $-1.24(1.96)$ & 0.528 & 71 & $-0.21(-0.86,0.44)$ & $0.21(-0.44,0.87)$ \\
\hline & & Sedentary $^{d}$ & $2.94(1.97)$ & 0.137 & 56 & $0.41(-0.13,0.94)$ & $-0.42(-0.98,0.13)$ \\
\hline & & LPA & $-1.88(0.91)$ & 0.041 & 18 & $-0.18(-0.35,-0.01)$ & $0.19(0.01,0.38)$ \\
\hline & & MVPA & $0.18(0.27)$ & 0.510 & 0.1 & $0.01(-0.03,0.06)$ & $-0.02(-0.07,0.03)$ \\
\hline \multirow[t]{4}{*}{$2(n=158)$} & $15.1(4.9)$ & Sleep & $-0.07(2.26)$ & 0.977 & 69 & $-0.01(-0.74,0.72)$ & $0.01(-0.73,0.75)$ \\
\hline & & Sedentary & $0.39(2.09)$ & 0.852 & 46 & $0.05(-0.46,0.56)$ & $-0.05(-0.59,0.49)$ \\
\hline & & LPA & $0.53(2.06)$ & 0.796 & 28 & $0.06(-0.36,0.48)$ & $-0.06(-0.51,0.39)$ \\
\hline & & MVPA & $-0.86(0.49)$ & 0.084 & 0.6 & $-0.07(-0.15,0.01)$ & $0.08(-0.01,0.17)$ \\
\hline \multirow[t]{4}{*}{$3.5(n=179)$} & $15.5(5.0)$ & Sleep & $-0.05(3.59)$ & 0.988 & 65 & $-0.01(-1.12,1.10)$ & $0.01(-1.12,1.14)$ \\
\hline & & Sedentary & $-0.54(2.94)$ & 0.856 & 47 & $-0.07(-0.80,0.66)$ & $0.07(-0.69,0.84)$ \\
\hline & & LPA & $1.94(2.10)$ & 0.357 & 29 & $0.20(-0.23,0.64)$ & $-0.22(-0.68,0.25)$ \\
\hline & & MVPA & $-1.35(0.82)$ & 0.102 & 2 & $-0.11(-0.25,0.02)$ & $0.13(-0.02,0.27)$ \\
\hline \multirow[t]{4}{*}{$5(n=213)$} & $15.7(4.8)$ & Sleep & $-0.29(3.36)$ & 0.932 & 64 & $-0.04(-1.06,0.97)$ & $0.04(-0.99,1.08)$ \\
\hline & & Sedentary & $0.47(2.50)$ & 0.851 & 49 & $0.06(-0.57,0.69)$ & $-0.06(-0.72,0.60)$ \\
\hline & & LPA & $0.41(1.86)$ & 0.825 & 28 & $0.04(-0.34,0.42)$ & $-0.05(-0.46,0.36)$ \\
\hline & & MVPA & $-0.60(0.77)$ & 0.438 & 3 & $-0.05(-0.18,0.08)$ & $0.06(-0.08,0.20)$ \\
\hline
\end{tabular}

LPA is light physical activity, MVPA is moderate-to-vigorous physical activity

${ }^{a}$ Adjusted for randomised group, sex, primiparous, maternal education, household deprivation, ethnicity and maternal BMI. Analyses at 1 year of age further adjusted for whether the child was walking unaided or not walking

${ }^{b}$ Coefficient for the first isometric log ratio (ilr) co-ordinate, which is proportional to the log ratio of the behavior and the geometric mean of the remaining behaviors; adjusted for remaining ilr co-ordinates

cAll sleep estimates include naps where relevant

${ }^{\mathrm{d}}$ All sedentary estimates include wake after sleep onset

Bolded values indicate $P<0.05$

exception (sleep negatively impacting on BMD at 5 years of age in cross-sectional analyses), whereas sedentary time at one year of age was positively, and time at 5 years of age negatively, associated with bone outcomes at 5 years. Our findings agree with recent reviews that have highlighted little effect of sedentary time or sleep on bone in young children [46, 47]. It is feasible that stronger relationships might be observed in older children given differences in bone accrual with maturation; children undergoing puberty gain bone more rapidly [48], perhaps allowing greater influence of relevant behaviors such as sleep and physical activity.

Our study has several strengths including repeated assessment of measures of interest, the use of 24-h accelerometry rather than a combination of approaches to measure all behaviors of interest, and the inclusion of DXA, allowing investigation of relationships with body composition and bone health, as well as BMI. However, our study also has some limitations. Because we were interested in sleep and how it might impact on other components of the day as well as more broad relationships with health, each 24-h 'day' is calculated as the time the child woke up on day 1 until the time they woke up on day 2 (and so on). This means that the length of each day varies both within and between participants, requiring all data to be scaled to $24 \mathrm{~h}$ periods. However, this method should more appropriately capture the co-dependence of the sleep and activity components with repeated days of measurement and it is the structure of the day, not the actual time, that is relevant [7]. Second, difficulty remains in determining the most appropriate cutpoints to denote different intensities of activity, particularly across different age groups [42]. Commonly accepted cutpoints for the Actical accelerometer do not exist for children spanning the age range in this study ( 1 to 5 years), which is problematic given the rapid development that occurs over this time [42]. We chose cutpoints from a variety of sources [33, 34] and applied them across the age range to allow comparisons by age, but this may have introduced some error, particularly in those under three years of age and for determining MVPA. These cutpoints were derived using an epoch length of $15 \mathrm{~s}$, which may be too long to correctly capture all of the brief bouts of MVPA that inherently occur via the intermittent nature of play in very young children $[49,50]$. As others have shown, shorter epochs ( $5 \mathrm{~s}$ ) provide improved estimates of sedentary behavior and physical activity in 2-3 year old children 
Table 7 Predictive and concurrent relationships between sleep and activity components at 1, 2, 3.5 and 5 years of age and total body minus head BMD $\left(\mathrm{g} / \mathrm{cm}^{2}\right)$ at 5 years ${ }^{\text {a }}$

\begin{tabular}{|c|c|c|c|c|c|c|c|}
\hline Age (years) & $\begin{array}{l}\text { Mean BMD (SD) } \\
\text { at } 5 \text { years of age } \\
\left(\mathrm{g} / \mathrm{cm}^{2}\right)\end{array}$ & Component & $\begin{array}{l}\text { Regression } \\
\text { coefficient }^{\text {b }} \text { (SE) }\end{array}$ & $p$-value & $\begin{array}{l}10 \% \text { relative } \\
\text { difference in } \\
\text { minutes }\end{array}$ & $\begin{array}{l}\text { Estimated difference }(95 \% \mathrm{Cl}) \\
\text { in } \mathrm{BMD} \text { with a } 10 \% \text { increase } \\
\text { in component }\end{array}$ & $\begin{array}{l}\text { Estimated difference }(95 \% \mathrm{Cl}) \\
\text { in } \mathrm{BMD} \text { with a } 10 \% \text { decrease } \\
\text { in component }\end{array}$ \\
\hline \multirow[t]{4}{*}{$1(n=229)$} & \multirow[t]{4}{*}{$0.608(0.037)$} & Sleep ${ }^{c}$ & $0.010(0.015)$ & 0.516 & 71 & $0.002(-0.003,0.007)$ & $-0.002(-0.007,0.003)$ \\
\hline & & Sedentary $^{d}$ & $-0.032(0.015)$ & 0.029 & 56 & $-0.004(-0.008,-0.0005)$ & $0.005(0.0005,0.009)$ \\
\hline & & LPA & $0.024(0.007)$ & 0.001 & 18 & $0.002(0.001,0.004)$ & $-0.002(-0.004,-0.001)$ \\
\hline & & MVPA & $-0.001(0.002)$ & 0.632 & 0.1 & $0.000(-0.0004,0.0002)$ & $0.000(-0.0003,0.0005)$ \\
\hline \multirow[t]{4}{*}{$2(n=158)$} & \multirow[t]{4}{*}{$0.609(0.036)$} & Sleep & $-0.003(0.015)$ & 0.839 & 69 & $-0.001(-0.005,0.004)$ & $0.001(-0.004,0.005)$ \\
\hline & & Sedentary & $-0.010(0.014)$ & 0.465 & 46 & $-0.001(-0.005,0.002)$ & $0.001(-0.002,0.005)$ \\
\hline & & LPA & $0.002(0.014)$ & 0.904 & 28 & $0.000(-0.003,0.003)$ & $0.000(-0.003,0.003)$ \\
\hline & & MVPA & $0.012(0.003)$ & 0.001 & 0.6 & $0.001(0.0004,0.0015)$ & $-0.001(-0.0017,-0.0005)$ \\
\hline \multirow[t]{4}{*}{$3.5(n=178)$} & \multirow[t]{4}{*}{$0.607(0.037)$} & Sleep & $-0.021(0.027)$ & 0.426 & 65 & $-0.003(-0.012,0.005)$ & $0.003(-0.005,0.012)$ \\
\hline & & Sedentary & $-0.007(0.021)$ & 0.751 & 47 & $-0.001(-0.006,0.004)$ & $0.001(-0.005,0.006)$ \\
\hline & & LPA & $0.015(0.016)$ & 0.351 & 29 & $0.002(-0.002,0.005)$ & $-0.002(-0.005,0.002)$ \\
\hline & & MVPA & $0.014(0.006)$ & 0.023 & 2 & $0.001(0.0002,0.0021)$ & $-0.001(-0.0023,-0.0002)$ \\
\hline \multirow[t]{4}{*}{$5(n=213)$} & \multirow[t]{4}{*}{$0.609(0.037)$} & Sleep & $-0.054(0.025)$ & 0.031 & 64 & $-0.008(-0.0008,-0.0159)$ & $0.009(0.0008,0.0162)$ \\
\hline & & Sedentary & $0.036(0.019)$ & 0.055 & 49 & $0.005(-0.0001,0.0092)$ & $-0.005(-0.0097,0.0001)$ \\
\hline & & LPA & $-0.001(0.014)$ & 0.953 & 28 & $0.000(-0.003,0.003)$ & $0.000(-0.003,0.003)$ \\
\hline & & MVPA & $0.019(0.006)$ & 0.001 & 3 & $0.002(0.0007,0.0026)$ & $-0.002(-0.0028,-0.0007)$ \\
\hline
\end{tabular}

LPA is light physical activity, MVPA is moderate-to-vigorous physical activity

${ }^{a}$ Adjusted for randomised group, sex, primiparous, maternal education, household deprivation, ethnicity and maternal BMI. Analyses at 1 year of age further adjusted for whether the child was walking unaided or not walking

${ }^{b}$ Coefficient for the first isometric log ratio (ilr) co-ordinate, which is proportional to the log ratio of the behavior and the geometric mean of the remaining behaviors; adjusted for remaining ilr co-ordinates

'All sleep estimates include naps where relevant

${ }^{\mathrm{d}}$ All sedentary estimates include wake after sleep onset

Bolded values indicate $P<0.05$

using the ActiGraph accelerometer [51]. The cut-point used to denote MVPA in the current study $(\geq 698$ counts per $15 \mathrm{~s}$ ) was based on validation studies in toddlers and pre-schoolers [32, 33] but is also considerably higher than other validation studies published subsequently [34, 52]. Our findings regarding MVPA should thus be viewed with caution, particularly given others have stressed the difficulties of assessing MVPA in those under three years of age [51]. In our analyses, we controlled for sex rather than undertaking sex-specific analyses so that our sample sizes, and therefore power, were not reduced. This may be a limitation given that differences in physical activity may be apparent even at this young age. Sensitivity analyses did demonstrate that the significant cross-sectional relationships observed between compositional time use and BMI z-score at 3.5 years of age were only apparent in girls (data not shown). However, because of the lack of significant relationships observed generally in our study, and the small effect sizes in those that were significant, we did not complete any remaining sex-specific analyses. Although several methods have been suggested for adjusting BMC or BMD for factors such as body size, pubertal stage, skeletal maturity and body composition, there is currently no consensus on the optimum method to use [53]. The International Society for Clinical Densitometry suggests adjusting for height is appropriate in children with chronic diseases in whom poor growth and delayed puberty adversely affect bone size [54]. As our participants were healthy, and fewer than $5 \%$ of the cohort had height Z-scores $<3$ rd or $>97$ th percentile, we considered that adjusting for BMI Z-score was more appropriate. We only had valid accelerometry data suitable for these analyses from 27 to $45 \%$ of the original cohort at any one time, and only 139 children (17.3\% of the original cohort) had accelerometry data at all 4 time points, introducing the possibility that our results might not generalise to the population as a whole. However, comparison of demographic variables at baseline in those who did and did not supply data showed relatively little difference between the groups. Finally, it was not possible to determine the effect of compositional time use on body composition at 5 years of age with adjustment for body composition at a younger age as we only had DXA measurements at 5 years of age. Adjustment for other variables related to body composition such as dietary intake and screen use was also not possible as consistent measures of these variables were not available throughout the project. 
Table 8 Predictive relationships between sleep and activity components at 1,2 and 3.5 years of age and total body minus head BMC (g) at 5 years ${ }^{a}$

\begin{tabular}{|c|c|c|c|c|c|c|c|}
\hline Age (years) & $\begin{array}{l}\text { Mean BMC (SD) at } \\
5 \text { years of age }(\mathrm{g})\end{array}$ & Component & $\begin{array}{l}\text { Regression } \\
\text { coefficient } \\
\text { (SE) }\end{array}$ & $p$-value & $\begin{array}{l}10 \% \text { relative } \\
\text { difference in } \\
\text { minutes }\end{array}$ & $\begin{array}{l}\text { Estimated difference }(95 \% \mathrm{Cl}) \\
\text { in BMC with a } 10 \% \text { increase } \\
\text { in component }\end{array}$ & $\begin{array}{l}\text { Estimated difference }(95 \% \mathrm{Cl}) \\
\text { in } \mathrm{BMC} \text { with a } 10 \% \text { decrease } \\
\text { in component }\end{array}$ \\
\hline \multirow[t]{4}{*}{$1(n=229)$} & $380(74)$ & Sleep ${ }^{c}$ & $49.3(27.5)$ & 0.075 & 71 & $8.4(-0.8,17.6)$ & $-8.4(-17.7,0.8)$ \\
\hline & & Sedentary ${ }^{d}$ & $-88.7(27.7)$ & 0.002 & 56 & $-12.3(-19.8,-4.8)$ & $12.8(5.0,20.6)$ \\
\hline & & LPA & $42.6(12.8)$ & 0.001 & 18 & $4.0(1.7,6.4)$ & $-4.4(-7.0,-1.8)$ \\
\hline & & MVPA & $-3.2(3.8)$ & 0.400 & 0.1 & $-0.3(-0.9,0.4)$ & $0.3(-0.4,1.0)$ \\
\hline \multirow[t]{4}{*}{$2(n=158)$} & $383(73)$ & Sleep & $19.6(28.2)$ & 0.488 & 69 & $3.2(-5.9,12.4)$ & $-3.3(-12.5,6.0)$ \\
\hline & & Sedentary & $-46.6(26.2)$ & 0.077 & 46 & $-5.8(-12.2,0.6)$ & $6.1(-0.6,12.9)$ \\
\hline & & LPA & $3.1(25.7)$ & 0.904 & 28 & $0.3(-4.9,5.6)$ & $-0.4(-6.0,5.3)$ \\
\hline & & MVPA & $23.9(6.1)$ & $<0.001$ & 0.6 & $2.0(1.0,3.0)$ & $-2.2(-3.3,-1.1)$ \\
\hline \multirow[t]{4}{*}{$3.5(n=178)$} & $379(74)$ & Sleep & $-16.9(50.4)$ & 0.738 & 65 & $-2.7(-18.3,12.9)$ & $2.7(-13.2,18.6)$ \\
\hline & & Sedentary & $-26.0(40.5)$ & 0.522 & 47 & $-3.3(-13.3,6.7)$ & $3.5(-7.1,14.0)$ \\
\hline & & LPA & $16.9(29.4)$ & 0.565 & 29 & $1.8(-4.3,7.8)$ & $-1.9(-8.4,4.6)$ \\
\hline & & MVPA & $26.0(11.1)$ & 0.021 & 2 & $2.2(0.3,4.0)$ & $-2.4(-4.4,-0.4)$ \\
\hline \multirow[t]{4}{*}{$5(n=213)$} & $383(73)$ & Sleep & $-93.5(47.7)$ & 0.051 & 64 & $-14.4(-28.8,0.005)$ & $14.7(-0.006,29.4)$ \\
\hline & & Sedentary & $75.1(35.4)$ & 0.035 & 49 & $9.6(0.7,18.5)$ & $-10.1(-19.5,-0.8)$ \\
\hline & & LPA & $-19.6(26.4)$ & 0.458 & 28 & $-2.0(-7.4,3.3)$ & $2.2(-3.6,8.0)$ \\
\hline & & MVPA & $38.0(10.9)$ & 0.001 & 3 & $3.2(1.4,5.0)$ & $-3.5(-5.5,-1.6)$ \\
\hline
\end{tabular}

LPA is light physical activity, MVPA is moderate-to-vigorous physical activity

${ }^{a}$ Adjusted for randomised group, sex, primiparous, maternal education, household deprivation, ethnicity and maternal BMI. Analyses at 1 year of age further adjusted for whether the child was walking unaided or not walking

${ }^{b}$ Coefficient for the first isometric log ratio (ilr) co-ordinate, which is proportional to the log ratio of the behavior and the geometric mean of the remaining behaviors; adjusted for remaining ilr co-ordinates

${ }^{\mathrm{c}}$ All sleep estimates include naps where relevant

${ }^{\mathrm{d}}$ All sedentary estimates include wake after sleep onset

Bolded values indicate $P<0.05$

\section{Conclusions}

Considerable changes in time use occur across the 24-h day from infancy to 5 years of age. However, how these young children used their time showed few significant relationships with body composition and bone health at this young age using analyses appropriately accounting for compositional time use.

\section{Additional file}

Additional file 1: Table S1. Variation matrices ${ }^{1}$ between sleep and activity components at 1, 2, 3.5 and 5 years of age. (DOCX $28 \mathrm{~kb}$ )

\section{Abbreviations}

BMC: Bone mineral content; BMD: Bone mineral density; BMl: Body mass index; DXA: Dual-energy x-ray absorptiometry; LPA: Light physical activity; MVPA: Moderate-to-vigorous physical activity; TBLH: Total body less head; WASO: Wake after sleep onset

\section{Acknowledgements}

We are grateful to all the families who participated in the POI study, and to the POI research staff in the Departments of Women's and Children's Health, and Medicine at the University of Otago.

\section{Funding}

The POI Study was funded by the Health Research Council of New

Zealand $(08 / 374,12 / 281,12 / 310)$ and the Southern District Health Board.
RWT is supported by a Fellowship from Karitane Products Society. The funding source had no role in the design and conduct of the study; collection, management, analysis, or interpretation of the data; preparation, review, or approval of the manuscript; or the decision to submit the manuscript for publication.

\section{Availability of data and materials}

The datasets used and/or analysed during the current study are available from the corresponding author on reasonable request.

\section{Authors' contributions}

RWT and BJT are Principal Investigators of $\mathrm{POI}$ and designed the overall project along with BCG, AMH, ARG, and JL; RWT conceived the idea for this study; JL, RS, and MH conducted the research; KMJ undertook the accelerometry and DXA analyses; JJH designed and undertook the statistical analyses; RWT wrote the first and subsequent drafts of the manuscript and all authors critically revised the manuscript for important intellectual content. RWT had primary responsibility for final content. All authors read and approved the final manuscript.

\section{Ethics approval and consent to participate}

The original intervention was approved by the Lower South Ethics Committee (LRS/12/08/063) and the follow-up study by the University of Otago Human Ethics Committee (12/274). Written informed consent was obtained from the parent/guardian of all child participants.

Consent for publication

Not applicable.

\section{Competing interests}

The authors declare that they have no competing interests. 


\section{Publisher's Note}

Springer Nature remains neutral with regard to jurisdictional claims in published maps and institutional affiliations.

\section{Author details}

'Departments of Medicine, Dunedin School of Medicine, University of Otago, PO Box 56, Dunedin, New Zealand. 'Departments of Women's and Children's Health, Dunedin School of Medicine, University of Otago, Dunedin, New Zealand. ${ }^{3}$ Departments of Human Nutrition, Dunedin School of Medicine, University of Otago, Dunedin, New Zealand. ${ }^{4}$ Departments of Biostatistics Unit, Dunedin School of Medicine, University of Otago, Dunedin, New Zealand. ${ }^{5}$ Departments of Office of the Dean, Dunedin School of Medicine, University of Otago, Dunedin, New Zealand.

Received: 26 July 2018 Accepted: 14 November 2018 Published online: 26 November 2018

\section{References}

1. Carter PJ, Taylor BJ, Williams SM, Taylor RW. A longitudinal analysis of sleep in relation to BMI and body fat in children: the FLAME study. BMJ. 2011;342:d2717.

2. Fatima Y, Doi SAR, Mamun AA. Longitudinal impact of sleep on overweight and obesity in children and adolescents: a systematic review and biasadjusted meta-analysis. Obes Rev. 2015;16:137-49.

3. Saunders TJ, Gray CE, Poitras VJ, Chaput JP, Janssen I, Katzmarzyk PT, Olds TS, Connor Gorbor S, Kho ME, Sampson M, et al. Combinations of physical activity, sedentary behaviour and sleep: relationships with health indicators in school-aged children and youth. Appl Phys Nutr Metab. 2016:41:S283-93.

4. Williams SM, Taylor BJ, Taylor RW. Do more active children sleep more? A repeated cross-sectional analysis using accelerometry. PLoS One. 2014;Apri 02. https://doi.org/10.1371/journal.pone.0093117.

5. Chaput J-P, Saunders TJ, Carson V. Interactions between sleep, movement and other non- movement behaviours in the pathogenesis of childhood obesity. Obes Rev. 2017:18:7-14.

6. Pedišić Z. Measurement issues and poor adjustments for physical activity and sleep undermine sedentary behaviour research - the focus should shift to the balance between sleep, sedentary behaviour, standing and activity. Kinesiology. 2014:46:135-46.

7. Chastain SF, Palarea-Albaladejo J, Dontje ML, Skelton DA. Combined effects of time spent in physical activity, sedentary be- haviors and sleep on obesity and cardio-metabolic health markers: a novel compositional data analysis approach. PLoS One. 2015:10:e0139984.

8. Tremblay MS, Carson V, Chaput JP, Adamo KB, Aubert S, Choquette L, et al: Canadian 24-hour movement guidelines for the early years ( $0-4$ years): an integration of physical activity, sedentary behavior, and sleep. 2017.

9. Okely AD, Ghersi D, Hesketh KD, Santos R, Loughran SP, Cliff DP, Shilton T, Grant D, Jones RA, Stanley RM, et al. A collaborative approach to adopting/ adapting guidelines - the Australian 24-hour movement guidelines for the early years (birth to 5 years: an integration of physical activity, sedentary behavior, and sleep). BMC Pub Health. 2017;17:869.

10. Carson V, Tremblay MS, Chastin SFM. Cross-sectional associations between sleep duration, sedentary time, physical activity, and adiposity indicators among Canadian preschool-aged children using compositional analyses. BMC Pub Health. 2017:17:848.

11. Fairclough SJ, Dumuid D, Taylor S, Curry W, McGrane B, Stratton G, Maher C, Olds TS. Fitness, fatness and the reallocation of time between children's daily movement behaviours: an analysis of compositional data. Int J Behav Nutr Phys Act. 2017;14:64.

12. Dumuid D, Stanford TE, Martin-Fernandez J-A, Pedisic Z, Maher CA, Lewis LK, Hron K, Katzmarzyk PT, Chaput JP, Fogelholm M, et al. Compositional data analysis for physical activity, sedentary time and sleep research; 2017. https://doi.org/10.1177/09062280217710835.

13. del Pozo-Cruz B, Gant N, del Pozo-Cruz J, Maddison R. Relationships between sleep duration, physical activity and body mass index in young new Zealanders; an isotemporal substitution analysis. PLoS One. 2017;12:e0184472

14. Taylor RW, Williams SM, Farmer VL, Taylor BJ: The stability of sleep patterns in children 3 to 7 years of age. 2015;166:697-702.

15. Hesketh KD, Downing KL, Campbell K, Crawford D, Salmon J, Hnatiuk JA Proportion of infants meeting the Australian 24-hour movement guidelines for the early years: data from the Melbourne InFANT program. BMC Pub Health. 2017;17:856.
16. Lee E-Y, Hesketh KD, Hunter S, Kuzik N, Rhodes RE, Rinaldi CM, Spence JC, Carson V. Meeting new Canadian 24-hour movement guidelines for the early years and associations with adiposity among toddlers living in Edmonton, Canada. BMC Pub Health. 2017:17:840.

17. Santos R, Zhang Z, Pereira JR, Sousa-Sa E, Cliff DP, Okely AD. Compliance with the Australian 24-hour movement guidelines for the early years: associations with weight status. BMC Pub Health. 2017;17:867.

18. Moir C, Meredith-Jones K, Taylor BJ, Gray AR, Heath A-LM, Dale K, Galland BC, Lawrence JA, Sayers RM, Taylor RW. Early intervention to encourage physical activity in infants and toddlers. an RCT Med Sci Sports Exerc. 2016; 48:2246-453.

19. Taylor BJ, Gray AR, Galland BC, Heath A-L, Lawrence JA, Sayers RM, Cameron SL, Hanna M, Dale K, Coppell KJ, Taylor RW: Targeting sleep, food, and activity in infants for obesity prevention: an RCT. 2017;139:e20162037.

20. Taylor RW, Gray AR, Heath A-LM, Galland BC, Lawrence JA, Sayers RM Healey D, Tannock GW, Meredith-Jones K, Hanna M, et al. Sleep, nutrition and physical activity interventions to prevent obesity in infancy: Follow-up of the POI randomized controlled trial at 3.5 \& 5 years. Am J Clin Nutr. 2018; 108:228-36.

21. Taylor BJ, Heath A-L, Galland BC, Gray AR, Lawrence JA, Sayers RM, Dale K, Coppell KJ, Taylor RW. Prevention of overweight in infancy (POI.Nz) study: a randomised controlled trial of sleep, food and activity interventions for preventing overweight from birth. BMC Public Health. 2011;11:942.

22. Salmond C, Crampton P, Atkinson J. NZDep index of deprivation: user's manual. Wellington. New Zealand: Department of Public Health, University of Otago; 2007.

23. de Onis M, Onyango AW, Van den Broeck J, Chumlea WC, Martorell R. Measurement and standardization protocols for anthropometry used in the construction of a new international growth reference. Food Nutr Bull. 2004; 25:S27-36.

24. Taylor RW, Heath A-LM, Galland BC, Cameron SL, Lawrence JA, Gray AR, Tannock GW, Lawley B, Healey D, Sayers RM, et al. Three-year follow-up of a randomised controlled trial to reduce excessive weight gain in the first two years of life: protocol for the POI follow-up study. BMC Public Health. 2016;16:771.

25. World Health Organization. WHO Child Growth Standards based on length/ height, weight and age. 2006:450:76-85.

26. Meredith-Jones K, Williams SM, Galland BC, Kennedy G. Taylor RW: 24hr accelerometry: Impact of sleep-screening methods on estimates of physical activity and sedentary time. 2016;34:679-85.

27. Galland BC, Meredith-Jones K, Gray A, Taylor BJ, Taylor RW. Criteria for nap identification in infants and young children using 24-hour actigraphy and agreement with parental diary. Sleep Med. 2016;19:85-92.

28. Esliger DW, Copeland $\lrcorner$, Barnes JD, Tremblay MS. Standardizing and optimizing the use of accelerometer data for free-living physical activity monitoring. J Phys Act Health. 2005;2:366-83.

29. Hnatiuk JA, Ridgers ND, Salmon J, Campbell KJ, McCallum Z, Hesketh KD. Physical activity levels and patterns of 19-month-old children. Med Sci Sports Exerc. 2012;44:1715-20.

30. Rich C, Geraci M, Griffiths L, Sera F, Dezateux C, Cortina-Borja M. Quality control methods in accelerometer data processing: defining minimium wear time. PLoS One. 2013:8:e67206.

31. Meredith-Jones K, Haszard JJ, Moir C, Heath A-L, Galland BC, Taylor BJ, Gray AR, Sayers RM, Taylor RW: Physical activity and inactivity trajectories associated with body composition in preschoolers 2018;doi: https://doi.org/10.1038/s41366-018-0058-5.

32. Pfeiffer KA, Mclver KL, Dowda M, Almeida MJ, Pate RR. Validation and calibration of the Actical accelerometer in preschool children. Med Sc Sports Exerc. 2006;38:152-7.

33. Trost SG, Loprinzi P, Fees B. Identification of accelerometer cut-points for toddlers. In International Society for Behavioural Nutrition and Physical Activity; Minneapolis. 2010:165-6.

34. Adolph A, Puyau M, Vohra F, Nicklas T, Zakeri I, Butte NF. Validation of uniaxial and triaxial accelerometers for the assessment of physical activity in children. J Phys Act Health. 2012;9:944-53.

35. Goulding A, Taylor RW, Jones IE, Lewis-Barned NJ, Williams SM. Body composition of 4 and 5-year old New Zealand girls: a DXA study of initial adiposity and subsequent 4-year fat change. Int J Obes. 2003;27:410-5.

36. Crabtree NJ, Arabi A, Bachrach LK, Fewtrell M, Fuleihan GE, Kecskemethy $\mathrm{HH}$, Jaworski M, Gordon CM. Dual-energy X-ray absorptiometry interpretation and reporting in children and adolescents: the revised 2013 ISCD pediatric official positions. J Clin Densitom. 2014;17:225-42. 
37. Poitras VJ, Gray CE, Borghese MM, Carson V, Chaput JP, Janssen I, Katzmarzyk PT, Pate RR, Gorber SC, Kho ME, et al. Systematic review of the relationships between objectively measured physical activity and health indicators in school-aged children and youth. Appl Physiol Nutr Metab. 2016;41:S197-239.

38. Chaput JP, Gray CE, Poitras VJ, Carson V, Gruber R, Birken CS, MacLean JE, Aubert S, Sampson M, Tremblay MS. Systematic review of the relationships between sleep duration and health indicators in the early years (0-4 years). BMC Pub Health. 2017;17:855.

39. Carson V, Lee E-Y, Hewitt L, Jennings C, Hunter S, Kuzik N, Stearns JA, Unrau SP, Poitras VJ, Gray C, et al. Systematic review of the relationships between physical activity and health indicators in the early years (aged 0-4 years). BMC Pub Health. 2017;17:854

40. Guyatt GH, Oxman AD, Schunemann HJ, Tugwell P, Knottnerus A. GRADE guidelines: a new series of articles in the journal of clinical epidemiology. J Clin Epidemiol. 2011;64:380-2.

41. Dumuid D, Stanford TE, Pedisic Z, Maher C, Lewis LK, Martin-Fernandez J-A, Katzmarzyk PT, Chaput JP, Fogelholm M, Standage M, et al. Adiposity and the isotemporal subsitution of physical activity, sedentary time and sleep among school-aged children: a compositional data analysis approach. BMC Public Health. 2018;18:311.

42. Migueles JH, Cadenas-Sanchez C, Ekelund U, Nystrom CD, Mora-Gonzalez J, Lof M, Labayen I, Ruiz JR, Ortega FB. Accelerometer data collection and processing criteria to assess physical activity and other outcomes: a systematic review and practical considerations. Sports Med. 2017;47:1821-45.

43. Collings PJ, Brage S, Bingham DD, Costa S, West J, McEachan RRC, Wright J, Barber SE. Physical activity, sedentary time, and fatness in a biethnic sample of young children. Med Sci Sports Exerc. 2017:49:930-8.

44. Garcia-Hermoso A, Saavedra JM, Ramirez-Velez R, Ekelund U, del Pozo-Cruz B. Reallocating sedentary time to moderate-to-vigorous physical activity but not to light-intensity physical activity is effective to reduce adiposity among youths: a systematic review and meta-analysis. Obes Rev. 2017;18:1088-95.

45. Timmons BW, LeBlanc AG, Carson V, Connor Gorbor S, Dillman C, Janssen I, Kho ME, Spence JC, Stearns JA, Tremblay MS. Systematic review of physical activity and health in the early years (aged 0-4 years). Appl Physiol Nutr Metab. 2012;37:773-92.

46. Casazza K. Hanks $L$ J, Fernandez JR. Shorter sleep may be a risk factor for impaired bone mass accrual in childhood. J Clin Densitom. 2011;14:453-7.

47. Jones $G$, Dwyer $T$. Bone mass in prepubertal children: gender differences and the role of physical activity and sunlight exposure. J Clin Endocrinol. 1998:83:4274-9.

48. Bailey DA, McKay HA, Mirwald RL, Crocker PR, Faulkner RA. A six-year longitudinal study of the relationship of physical activity to bone mineral accrual in growing children: the university of Saskatchewan bone mineral accrual study. J Bone Min Res. 1999;14:1672-9.

49. Cliff DP, Reilly JJ, Okely AD. Methodological considerations in using accelerometers to assess habitual physical activity in children aged 0-5 years. J Sci Med Sport. 2009;12:557-67.

50. Oliver M, Schofield G, Schulter PJ. Accelerometry to assess preschooler's free-play: issues with count thresholds and epoch durations. Meas Phys Educ Exerc Sci. 2009;13:181-90.

51. Costa S, Barber SE, Cameron N, Clemes SA. Calibration and validation of the ActiGraph GT3X+ in 2-3 year olds. J Sci Med Sport. 2014;17:617-22.

52. Janssen X, Cliff DP, Reilly JJ, Hinkley T, Jones R, Batterham M, Ekelund U, Brage S, Okely AD. Evaluation of Actical equations and thresholds to predict physical activity intensity in young children. Phys Act Health. 2015;33:498-506.

53. Zemel BS, Leonard MB, Kelly A, Lappe JM, Gilsanz V, Oberfield S, Mahboub S, Shepherd JA, Hangartner TN, Frederick MM, et al. Height adjustment in assessing dual energy $x$-ray absorptiometry measurements of bone mass and density in children. J Clin Endocrinol Metab. 2010;95:1265-73.

54. Gordon CM, Leonard MB, Zemel BS. 2013 pediatric position development conference: executive summary and reflections. J Clin Densitom. 2013;17:219-24.

Ready to submit your research? Choose BMC and benefit from:

- fast, convenient online submission

- thorough peer review by experienced researchers in your field

- rapid publication on acceptance

- support for research data, including large and complex data types

- gold Open Access which fosters wider collaboration and increased citations

- maximum visibility for your research: over $100 \mathrm{M}$ website views per year

At BMC, research is always in progress.

Learn more biomedcentral.com/submissions 